\title{
Recusa da vacinação em área urbana do norte de Portugal
}

\author{
Vaccine refusal in an urban area of northern Portugal
}

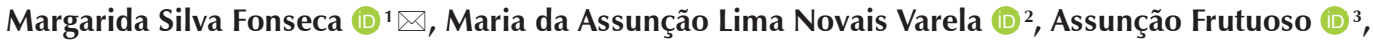 \\ Maria de Fátima Ferreira Ramos Pinto Monteiro ${ }^{4}$ \\ ' Centro Hospitalar do Tâmega e Sousa, Serviço de Pediatria e Neonatologia. Penafiel, Portugal. \\ 2 Agrupamento de Centros de Saúde do Porto Ocidental, Unidade de Saúde Pública. Porto, Portugal. \\ ${ }^{3}$ Administração Regional de Saúde do Norte, Departamento de Saúde Pública, Coordenação Regional do Programa Nacional de Vacinação. Porto, Portugal. \\ ${ }^{4}$ Agrupamento de Centros de Saúde do Porto Ocidental, Unidade de Recursos Partilhados, Departamento de Pediatria. Porto, Portugal.
}

Como citar este artigo (How to cite this article):

Fonseca MS, Varela MALN, Frutuoso A, Monteiro MFFRP. Recusa da vacinação em área urbana do norte de Portugal (Vaccine refusal in an urban area of northern Portugal). Sci Med. 2018;28(4):ID32152. DOI: 10.15448/1980-6108.2018.4.32152

\section{RESUMO}

OBJETIVOS: Conhecer o número de recusas vacinais e investigar os motivos de não adesão à vacinação pelos pais de crianças e adolescentes residentes numa área urbana do norte de Portugal.

MÉTODOS: Estudo transversal com amostra obtida dos registros de crianças/adolescentes até os 14 anos, inscritos em unidades de saúde de uma área metropolitana da cidade do Porto, Portugal, pertencentes aos agrupamentos de centros de saúde Porto Ocidental, Porto Oriental, Gaia, Gondomar e Matosinhos, cujos pais recusaram alguma vacina do Programa Nacional de Vacinação, no período de janeiro de 2009 a dezembro de 2015. A caraterização da amostra e os motivos de recusa vacinal foram estudados através da aplicação de um questionário aos pais. RESULTADOS: Foram identificados 150 casos de crianças/adolescentes aos quais os pais recusaram alguma vacina, numa população global de 103.406 crianças, resultando em uma taxa de recusa vacinal de $0,14 \%$. A maior taxa ocorreu no agrupamento de centros de saúde Porto Ocidental: 0,31\%. Entre os 150 casos, 86 pais aceitaram responder ao questionário, correspondendo a uma taxa de adesão de $64 \%$. A mediana da idade das crianças/adolescentes cujos pais recusaram a vacinação foi de sete anos, sendo a maioria saudável e sem problemas perinatais Todos os pais eram adultos, a maioria casados e do gênero feminino. A maioria tinha curso superior e encontrava-se em atividade profissional. $\mathrm{O}$ agrupamento de centros de saúde do Porto Ocidental foi aquele onde os pais que recusaram vacinas eram majoritariamente do sexo masculino, tinham nível acadêmico mais alto e eram mais ativos profissionalmente. A vacina com maior taxa de recusa foi a BCG, seguida da anti-papilomavírus humano e da vacina contra sarampo, caxumba e rubéola. Os quatro principiais motivos de recusa vacinal referidos pelos pais foram: "as vacinas não são uma prioridade", "as vacinas são pouco seguras", "indicação do médico assistente" e "receio de efeitos colaterais". O motivo "indicação do médico assistente" referiu-se, em todos os casos, à vacina BCG.

CONCLUSÕES: Nesta população foi pequena a taxa de recusa vacinal; no entanto, com base nos resultados obtidos, podem ser dirigidos esforços de intervenção junto às famílias, visando combater os argumentos sem base científica e obter uma adesão inequívoca ao Programa Nacional de Vacinação em Portugal.

DESCRITORES: recusa de vacinação; hesitação vacinal; programas de imunização; pediatria; criança; adolescente.

\section{ABSTRACT}

AIMS: To know the number of vaccine refusals and to investigate the reasons for non-compliance with vaccination by the parents of children and adolescents living in an urban area of northern Portugal.

METHODS: A cross-sectional study was carried out with a sample of children/adolescents up to the age of 14 enrolled in health units in a metropolitan area of Porto, Portugal, belonging to the health centers groupings of Porto Ocidental, Porto Oriental, Gaia, Gondomar and Matosinhos, whose parents refused any vaccination of the National Vaccination Program in the period from January 2009 to December 2015. We studied the characterization of the sample and the reasons for vaccine rejection through the application of a questionnaire to parents. RESULTS: We identified 150 cases of children/adolescents to whom the parents refused any vaccine, in a global population of 103,406 children, resulting in a vaccine refusal rate of $0.14 \%$. The highest rate occurred in the Porto Ocidental health centers grouping: $0.31 \%$. Among the 150 cases, 86 parents accepted to respond to the questionnaire, corresponding to an adhesion rate of $64 \%$. The median age of children/adolescents whose parents refused vaccination was seven years; most of them were healthy and had no perinatal problems. All parents were adults, mostly married and female. Most parents had a college degree and were professionally active. The Porto Oriental health centers grouping was the one where the parents who refused vaccines were mostly males, had a higher academic level and were more professionally active. The vaccine with the highest refusal rate was BCG, followed by anti-human papillomavirus and measles, mumps and rubella vaccine. The four main reasons for vaccination refusal by the parents were "vaccines are not a priority", "vaccines are not safe", "indication of the attending physician" and "fear of side effects." The reason for the "indication of the attending physician" was in all cases referred to the BCG vaccine.

CONCLUSIONS: In this population the vaccination refusal rate was low; however, based on the results obtained, intervention efforts can be directed towards the families, aiming at combating the arguments without scientific basis and obtaining an unequivocal adhesion to the National Vaccination Program in Portugal.

KEYWORDS: vaccination refusal; vaccine hesitancy; immunization programs; pediatrics; child; adolescent. 
Abreviaturas: ACeS, Agrupamento(s) de Centros de Saúde; BCG, bacilo Calmette-Guerin (vacina anti-tuberculose); DTPA, difteria, tétano e pertussis acelular; HIB, Haemophilus influenzae do tipo B; HPV, human papillomavirus (papilomavírus humano); MenC, meningococo C; OMS, Organização Mundial da Saúde; PNV, Programa Nacional de Vacinação; Pn13, vacina pneumocócica conjugada 13-valente; RV, recusa vacinal; SAGE, Strategic Advisory Group of Experts; VASPR, vacina contra sarampo, parotidite e rubéola; VHB, vírus da hepatite B; VIP, vacina inativada da poliomielite.

\section{INTRODUÇÃO}

A vacinação constitui uma das medidas mais eficientes na promoção da saúde pública e individual, evitando cerca de dois a três milhões de mortes anuais e conferindo proteção contra pelo menos 30 doenças infeciosas em nível mundial $[1,2]$. A erradicação mundial da varíola e a eliminação da poliomielite em quatro das grandes regiões consideradas pela Organização Mundial de Saúde (OMS) refletem a importância dos programas de vacinação. No entanto, as doenças infeciosas ainda representam uma ameaça significativa na Europa, tendo sido reportados em 2013 , cerca de 31.685 casos de sarampo e 39.367 casos de rubéola [3].

Em Portugal, o Programa Nacional de Vacinação (PNV) entrou em vigor em 1965, constituindo-se como um programa universal, gratuito e acessível a todas as pessoas [4]. Os esquemas aconselhados no PNV aplicam-se ao longo de toda da vida, por exemplo, com vacinas contra tétano e difteria para toda a vida e, mais recentemente, com a vacina contra a coqueluche (em Portugal, "tosse convulsiva") durante a gestação (Norma no 16/2016, atualizada em 31 de julho de 2017) [5].

Desde 2015, as vacinas do PNV protegem contra 13 doenças - tuberculose (vacina bacilo CalmetteGuerin - BCG); hepatite B (vacina conta o vírus da hepatite B - VHB); difteria, tétano, coqueluche (vacina difteria, tétano e pertussis acelular - DTPA); poliomielite (vacina inativada da poliomielite VIP); doença invasiva por Haemophilus influenzae do tipo b (HIB); sarampo, parotidite epidêmica, rubéola (vacina contra sarampo, caxumba e rubéola VASPR); doença invasiva por Neisseria meningitidis do serogrupo $\mathrm{C}$ (vacina contra o meningococo $\mathrm{C}$ MenC); infeções por papilomavírus humano (human papillomavirus - HPV) para as raparigas; e doença invasiva por Streptococcus pneumoniae (vacina pneumocócica conjugada 13-valente - Pn13). Essas vacinas encontram-se distribuídas em idades-chave de vacinação, com o objetivo de maximizar a sua eficácia (Parecer no 42/2013 da Comissão de Ética para a Saúde da Administração Regional de Saúde do Norte, Portugal).

Segundo dados do Departamento de Saúde Pública da Administração Regional de Saúde do Norte, as taxas de cobertura vacinal para as vacinas do PNV nos últimos anos em Portugal foram sempre superiores a 95\% [6]. É já reconhecido que o enorme sucesso da vacinação conduz à desvalorização do risco associado às doenças imunopreveníveis, podendo este fato gerar problemas na adesão à vacinação [7]. Surtos dessas doenças, incluindo sarampo, poliomielite e difteria, têm ressurgido em várias partes do mundo, como consequência de comunidades subvacinadas ou não vacinadas [8]. Por outro lado, preocupações com efeitos adversos e associação de vacinas com outras doenças, poderão também constituir um fator de não adesão à vacinação [10]. Também dúvidas e crenças de que as vacinas não têm a sua suposta eficácia, estão já apresentadas em estudos internacionalmente publicados sobre o tema da não vacinação ou hesitação em vacinar [9-16].

Apesar dos bons resultados alcançados pelos programas de vacinação, a recusa vacinal (RV) existe e é considerada um dos extremos da hesitação vacinal [12]. A hesitação em vacinar é uma entidade complexa, dinâmica e de amplo espectro, desde a aceitação em vacinar com incertezas até à recusa total inequívoca. A abordagem conceptual Model of determinants of vaccine hesitancy do grupo de trabalho sobre hesitação vacinal do Strategic Advisory Group of Experts (SAGE), da OMS, explica as influências da vacinação com base numa tríade epidemiológica, incluindo fatores contextuais, fatores individuais ou de grupo e questões inerentes à própria vacinação, por sua vez condicionada pelos determinantes fundamentais do "Modelo 3Cs" - complacência, conveniência e confiança $[9,14]$.

Sob o ponto de vista ético, em Portugal, é reconhecido ao indivíduo ou seu representante legal a possibilidade de consentir ou recusar qualquer procedimento médico, incluindo a vacinação - respeito pela autonomia do indivíduo [17]. Entretanto, em idade pediátrica, a RV deve ser alvo de uma profunda reflexão, pois essa decisão dos pais, além de condicionar a imunidade individual, poderá pôr em risco a saúde da comunidade (imunidade de grupo) e constituir um sério problema de saúde pública.

Em Portugal, poucos estudos abordam prevalência e fatores envolvidos na opção pela não vacinação 
em idade pediátrica. Cabe aos profissionais de saúde, principalmente aqueles envolvidos na área da vacinação, a responsabilidade da procura ativa desses dados, para um maior conhecimento sobre o tema e intervenção eficaz na comunidade. Por isso, cada RV deverá constituir uma janela de oportunidade para o esclarecimento de dúvidas e preocupações junto dos pais da criança/adolescente, elucidando-os e alertando-os para a sua real importância e tentando promover uma adesão total e inequívoca à vacinação. Os objetivos principais deste trabalho foram conhecer o número de RV das vacinas do PNV por pais de crianças e adolescentes e conhecer os motivos da RV, para poder informar, esclarecer, alertar e desmistificar dúvidas inerentes à vacinação, assim como fornecer dados para a elaboração de estratégias de intervenção adequadas ao tipo de população em estudo.

\section{MÉTODOS}

\section{Delineamento e seleção da amostra}

Este estudo transversal teve como base a seleção de um grupo etário que incluísse as idades chave de vacinação utilizadas pela Direção-Geral da Saúde portuguesa para avaliação anual do cumprimento do PNV (através de taxas de cobertura vacinal). A seleção do período temporal em análise pretendeu englobar a informação mais recente respetiva a cada recusa, assim como a inclusão das alterações ocorridas no PNV no período de estudo (substituição do PNV de 2006 pelo PNV de 2012, alteração do esquema vacinal contra infeções pelo HPV em 2014, introdução da Pn13 em 2015). Novos esquemas de vacinação/alterações ao PNV aplicados a partir de 01 de janeiro de 2016 já não foram assim incluídos neste trabalho.

O tipo e a dimensão da amostra foram feitos através de uma amostragem de conveniência, constituída pelo número total de pais com RV de crianças e adolescentes até os 14 anos de idade, ocorridas num período temporal de sete anos entre 01 de janeiro de 2009 e 31 de dezembro de 2015, inscritas nas unidades de saúde da área metropolitana da cidade do Porto, Portugal, pertencentes aos agrupamentos de centros de saúde (ACeS): Porto Ocidental, Porto Oriental, Gaia, Gondomar e Matosinhos. Os critérios de exclusão foram pais que recusaram participar no estudo/não obtenção de consentimento informado pelos mesmos; contraindicação formal à vacinação dos seus filhos; vacinas em atraso pelo PNV; e opção por plano de vacinação estrangeiro.

\section{Ética e aquisição de dados}

O estudo foi aprovado pela Comissão de Ética da Administração Regional de Saúde do Norte (Parecer no 119/2016). Os casos de RV pelos pais, constituindo a amostra em estudo, foram levantados e informados aos pesquisadores pelos enfermeiros responsáveis pela vacinação de cada unidade de saúde dos ACeS integrados no trabalho, após a autorização da Comissão de Ética e com o conhecimento da Direção Executiva e conselhos clínicos dos ACeS integrados no trabalho. As demais informações, referentes a cada caso de RV, foram obtidas por meio de um questionário dirigido aos pais, anônimo e confidencial, aplicado por contato telefônico pela investigadora principal, após obtenção do consentimento informado livre e esclarecido, prestado de forma oral. As entrevistas foram realizadas durante os meses de outubro, novembro e dezembro de 2016 .

O questionário foi construído com base em reuniões com médicos de saúde pública e outros profissionais envolvidos na área da vacinação, assim como estudos e revisões internacionais publicados sobre o tema da não vacinação [8-15]. Para aferição da validade e confiabilidade do questionário, foi realizado um ensaio piloto aplicado aleatoriamente a 10 dos pais que recusaram vacinação e que aceitaram participar no estudo. Desse ensaio resultaram algumas adequações na forma de colocação das perguntas, mas sem alterações no conteúdo. Os participantes no ensaio piloto foram excluídos da análise do presente estudo. No questionário foram levantados dados socioeconômicos, culturais, biológicos e educacionais da criança/adolescente e pais/família; vacinas recusadas e principais motivos.

Em relação às razões apontadas para a $\mathrm{RV}$, pela possibilidade de existência de mais do que um motivo de RV pelos pais, as respostas obtidas através da aplicação do questionário foram organizadas, além da frequência total de resposta (número de vezes apontadas), de acordo com a ordem de resposta dada pelos pais (primeira, segunda ou terceira).

\section{Análise dos dados}

O banco de dados e a análise estatística dos dados foram realizados, respectivamente, nos programas Microsoft Office Excel 2016 e IBM SPSS versão 23. Para avaliação de diferenças estatísticas nas variáveis contínuas foi utilizado o Teste Kruskal-Wallis e para as variáveis categóricas foi usado o Teste do Qui-quadrado de Pearson. O nível de significância adotado foi 5\%. 


\section{RESULTADOS}

Foram identificados 150 casos de crianças/ adolescentes cujos pais apresentaram RV em relação aos seus filhos, numa população global de 103.406 crianças inscritas nas unidades de saúde pertencentes aos vários $\mathrm{ACeS}$ da área metropolitana da cidade do Porto. Portanto, a taxa de RV nessa população foi de $0,14 \%$. A maioria dos casos de RV foi encontrada no ACeS Porto Ocidental (população alvo de 22.699 crianças/adolescentes inscritos, $72 \mathrm{RV} ; 0,31 \%$ ), seguindo-se do ACeS Matosinhos (24.176 inscritos, $39 \mathrm{RV}$; 0,16\%), Gondomar (21.595 inscritos, $17 \mathrm{RV}$; $0,07 \%$ ), Porto Oriental (14.440 inscritos, $18 \mathrm{RV}$; $0,12 \%$ ) e Gaia (20.496 inscritos, $4 \mathrm{RV} ; 0,19 \%$ ).

Foram contatados 150 pais com RV, sendo que 54 recusaram participar no estudo e 96 pais aceitaram responder ao questionário. Excluídos os 10 pais do ensaio piloto, foram incluídos 86 respondentes, correspondendo a uma taxa de adesão de $64 \%$.
Algumas das questões incluídas no questionário não foram respondidas por alguns pais, resultando assim em alguns itens com um número total inferior a 86 .

A mediana da idade das crianças/adolescentes cujos pais recusaram a vacinação foi de sete anos de idade (intervalo interquartil 1-14, mínima $=0$ máxima=14). Houve predomínio do sexo feminino e sem problemas de saúde associados. A maioria das mães dessas crianças/adolescentes havia feito pré-natal e não teve problemas no parto. Apenas crianças até os dois anos não frequentavam qualquer instituição de ensino, sendo que as restantes integravam o nível de ensino adequado à idade (Tabela 1).

Relativamente aos pais, todos eram adultos, na maioria casados e do gênero feminino. A nível acadêmico, a maioria tinha um curso superior e, a nível profissional, a maioria encontrava-se numa situação ativa. A maioria dos pais era saudável, o tipo de família predominante era o nuclear e algumas famílias seguiam uma dieta alimentar específica (Tabela 2).

Tabela 1. Caraterísticas de 86 crianças/adolescentes residentes em área urbana do norte de Portugal, cujos pais recusaram algum tipo de vacina do Programa Nacional de Vacinação. (Alguns itens não foram respondidos por todos os participantes).

\begin{tabular}{|c|c|c|}
\hline Caraterística da criança/adolescente & & $\begin{array}{c}\text { Total } \\
(n=86)\end{array}$ \\
\hline \multirow[t]{2}{*}{ Idade (anos) } & Mediana (intervalo interquartil) & $7(1 ; 14)$ \\
\hline & Mínima-máxima & $0-14$ \\
\hline \multirow[t]{2}{*}{ Sexo n (\%) } & Feminino & $54(63)$ \\
\hline & Masculino & $32(37)$ \\
\hline Pré-natal adequado n (\%) & & $84(98)$ \\
\hline \multirow[t]{2}{*}{ Local do parto $\mathrm{n}(\%)$} & Hospital & $84(98)$ \\
\hline & Casa & $2(2)$ \\
\hline \multirow[t]{5}{*}{ Problemas peri-parto n (\%) } & Não & $76(88)$ \\
\hline & Placentário & $1(1)$ \\
\hline & Ameaça de parto prematuro/parto prematuro & $5(6)$ \\
\hline & Restrição de crescimento intrauterino & $2(2)$ \\
\hline & Taquipneia transitória do recém-nascido & $2(2)$ \\
\hline \multirow[t]{8}{*}{ Doença atual n (\%) } & Não & $76(88)$ \\
\hline & Congênita & $1(1)$ \\
\hline & Endoócrina/metabólica & $2(2)$ \\
\hline & Respiratória & $3(4)$ \\
\hline & Neurológica & $1(1)$ \\
\hline & Mental/comportamental & $1(1)$ \\
\hline & Hematológica & $1(1)$ \\
\hline & Cutânea & $1(1)$ \\
\hline \multirow[t]{3}{*}{ Frequência a instituição de ensino, crianças até 2 anos de idade $n$ (\%) } & Não & $14(16)$ \\
\hline & Jardim de infância & $12(14)$ \\
\hline & Infantário (creche) & $8(9)$ \\
\hline Frequência a instituição de ensino, crianças com mais de 2 anos de idade $n$ (\%) & Nível de ensino adequado à idade & $52(100)$ \\
\hline
\end{tabular}


Tabela 2. Dados sociodemográficos relativos aos pais/família em 86 casos de recusa vacinal em crianças e adolescentes residentes em área urbana do norte de Portugal.

\begin{tabular}{|c|c|c|}
\hline Caraterística dos pais/família & & n (\%) \\
\hline \multirow[t]{2}{*}{ Gênero } & Feminino & $71(82)$ \\
\hline & Masculino & $15(18)$ \\
\hline \multirow[t]{3}{*}{ Estado civil } & Casado & $52(62)$ \\
\hline & Solteiro & $25(30)$ \\
\hline & Divorciado/Separado & $7(8)$ \\
\hline \multirow[t]{6}{*}{ Nível acadêmico (anos de estudo) } & Nenhum & 0 \\
\hline & $4 \% / 60$ ano & 0 \\
\hline & 9o ano & $4(5)$ \\
\hline & $12^{\circ}$ ano & $12(15)$ \\
\hline & Curso Profissional & $5(6)$ \\
\hline & Curso Superior & $62(75)$ \\
\hline \multirow[t]{2}{*}{ Situação profissional } & Ativo & $71(86)$ \\
\hline & Inativo & $12(14)$ \\
\hline \multirow[t]{4}{*}{ Tipo de família } & Nuclear & $66(86)$ \\
\hline & Alargada & $7(9)$ \\
\hline & Monoparental & $1(1)$ \\
\hline & Reconstruída & $3(4)$ \\
\hline \multirow[t]{4}{*}{ Rendimento familiar (ordenados mínimos) } & Até 1 & $10(12)$ \\
\hline & Entre 1 a 3 & $33(41)$ \\
\hline & De 3 a 6 & $25(31)$ \\
\hline & Mais do que 6 & $13(16)$ \\
\hline \multirow[t]{4}{*}{ Apoio econômico } & Não & $78(91)$ \\
\hline & Abono familiar & $6(7)$ \\
\hline & Subsídio escolar & $1(1)$ \\
\hline & Rendimento social de inserção & $1(1)$ \\
\hline \multirow[t]{2}{*}{ Tipo de habitação } & Casa & $22(26)$ \\
\hline & Apartamento & $61(74)$ \\
\hline \multirow[t]{3}{*}{ Doença } & Não & $83(97)$ \\
\hline & Tireoide & $1(1)$ \\
\hline & Hematológica & $2(2)$ \\
\hline \multirow[t]{3}{*}{ Outros filhos } & Nenhum & $23(29)$ \\
\hline & 1 & $37(46)$ \\
\hline & 2 & $20(25)$ \\
\hline \multirow[t]{5}{*}{ Tipo de Dieta } & Não Específica & $67(78)$ \\
\hline & Vegana & $6(7)$ \\
\hline & Ovolactovegetariana & $4(5)$ \\
\hline & Macrobiótica & $6(7)$ \\
\hline & Sem glúten e sem leite & $3(4)$ \\
\hline
\end{tabular}

Em relação às características dos pais, os itens que apresentaram diferença significativa entre os diversos ACeS estão representados na Tabela 3. No ACeS Porto Ocidental, no qual a taxa de RV foi maior, os pais que recusaram vacinas eram em maior porcentagem do sexo masculino, tinham nível acadêmico mais alto e havia maior porcentagem de ativos profissionalmente.

Relativamente às vacinas recusadas, aquela com maior taxa de recusa foi a BCG, seguida da vacina anti-HPV e da VASPR (Tabela 4).
Os quatro principiais motivos de RV referidos pelos pais, por ordem decrescente de frequência, foram: "as vacinas não são uma prioridade", "as vacinas são pouco seguras", "indicação do médico assistente" e "receio de efeitos colaterais". O motivo "indicação do médico assistente" constituiu aquele mais vezes indicado como primeira resposta, e referiram-se, em todos os casos, à vacina BCG (Tabela 5). 
Tabela 3. Características sociodemográficas dos pais/família que tiveram diferenças significativas $\left(p<0,05^{*}\right)$ na comparação entre os diversos Agrupamentos de Centros de Saúde de área metropolitana da cidade do Porto, Portugal.

\begin{tabular}{|c|c|c|c|c|c|c|c|}
\hline \multirow[b]{2}{*}{ Caraterística dos pais/família } & & \multirow{2}{*}{$\begin{array}{c}\text { Total } \\
(\mathbf{n}=86) \\
\mathrm{n}(\%)\end{array}$} & \multicolumn{5}{|c|}{ Agrupamento de Centros de Saúde } \\
\hline & & & $\begin{array}{c}\text { Ocidental } \\
(n=43) \\
n(\%)\end{array}$ & $\begin{array}{c}\text { Gondomar } \\
(\mathrm{n}=12) \\
\mathrm{n}(\%)\end{array}$ & $\begin{array}{c}\begin{array}{c}\text { Oriental } \\
(\mathrm{n}=11)\end{array} \\
\mathrm{n}(\%)\end{array}$ & $\begin{array}{c}\text { Gaia } \\
(n=3) \\
n(\%)\end{array}$ & $\begin{array}{c}\text { Matosinhos } \\
(\mathbf{n}=17) \\
\mathbf{n}(\%)\end{array}$ \\
\hline \multirow[t]{2}{*}{ Sexo } & M & $15(18)$ & $10(23)$ & 0 & $4(36)$ & 0 & $1(6)$ \\
\hline & $\mathrm{F}$ & $71(82)$ & $33(77)$ & $12(100)$ & $7(64)$ & $3(100)$ & $16(94)$ \\
\hline \multirow[t]{6}{*}{ Nível acadêmico (anos de estudo) } & Nenhum & 0 & 0 & 0 & 0 & 0 & 0 \\
\hline & $4^{\circ} / 6^{\circ}$ & 0 & 0 & 0 & 0 & 0 & 0 \\
\hline & 9o & $4(5)$ & $1(2)$ & $2(18)$ & $1(10)$ & 0 & 0 \\
\hline & $12^{\mathrm{o}}$ & $12(15)$ & $4(10)$ & $3(27)$ & 0 & 0 & $5(29)$ \\
\hline & Curso profissional & $5(6)$ & $1(2)$ & 0 & $3(30)$ & 0 & $1(6)$ \\
\hline & Curso superior & $62(75)$ & $36(86)$ & $6(55)$ & $6(60)$ & $3(100)$ & $11(65)$ \\
\hline \multirow[t]{2}{*}{ Situação profissional } & Ativo & $71(86)$ & $38(90)$ & $5(46)$ & $1(10)$ & $3(100)$ & $16(94)$ \\
\hline & Inativo & $12(14)$ & $4(10)$ & $6(54)$ & $9(90)$ & 0 & $1(6)$ \\
\hline
\end{tabular}

* Teste do Qui-quadrado.

Tabela 4. Número de recusas por tipo de vacina, pelos pais de crianças e adolescentes residentes em área urbana do norte de Portugal.

\begin{tabular}{lc}
\hline Vacina & $\begin{array}{c}\text { Número de } \\
\text { recusas }\end{array}$ \\
\hline Todas & 9 \\
BCG (tuberculose) & 31 \\
VHB (vacina contra hepatite B) & 10 \\
DTPA/HIB/VIP/VHB & 4 \\
DTPA/HIB/VIP & 10 \\
DTPA/HIB & 6 \\
\hline
\end{tabular}

\begin{tabular}{lc}
\hline Vacina & $\begin{array}{c}\text { Número de } \\
\text { recusas }\end{array}$ \\
\hline DTPA/VIP & 8 \\
Vacina contra meningococo C & 11 \\
VASPR (vacina contra sarampo, caxumba e rubéola) & 17 \\
TD (vacina contra tétano e difteria - tipo adulto) & 7 \\
HPV & 26 \\
\hline
\end{tabular}

DTPA: vacina contra difteria, tétano e coqueluche; HIB: vacina contra doença invasiva por Haemophilus influenzae tipo B; VIP: vacina contra poliomielite; HPV: vacina contra infeções por vírus do papiloma humano.

Tabela 5. Motivos de recusa vacinal referidos pelos pais por ordem de escolha, de acordo com a prioridade estabelecida.

\begin{tabular}{|c|c|c|c|c|c|}
\hline \multirow{2}{*}{ Motivo da recusa vacinal } & & \multirow{2}{*}{$\begin{array}{l}\text { Total de } \\
\text { escolhas } \\
(n=177)\end{array}$} & \multicolumn{3}{|c|}{ Ordem de escolha } \\
\hline & & & $\begin{array}{l}\text { 1- escolha } \\
(n=80)\end{array}$ & $\begin{array}{l}2^{\mathrm{a}} \text { escolha } \\
(\mathrm{n}=60)\end{array}$ & $\begin{array}{l}3^{\text {a }} \text { escolha } \\
(n=37)\end{array}$ \\
\hline A vacina não é prioridade & & 33 & 12 & 17 & 4 \\
\hline A vacina é pouco segura & & 32 & 8 & 11 & 13 \\
\hline Indicação do médico assistente* & & 31 & 29 & 2 & 0 \\
\hline Receio de efeitos colaterais & & 19 & 1 & 9 & 9 \\
\hline A vacina não é eficaz & & 13 & 3 & 6 & 4 \\
\hline Crenças pessoais & & 11 & 7 & 2 & 2 \\
\hline \multirow[t]{3}{*}{ Opção por outros métodos de prevenção de doenças } & Sim & 11 & & & \\
\hline & Suplementos nutricionais & 1 & 0 & 1 & 0 \\
\hline & Homeopatia & 10 & 4 & 4 & 2 \\
\hline \multirow[t]{3}{*}{ Informação negativa sobre vacinas na comunicação social } & Total & 10 & & & \\
\hline & Internet & 8 & 6 & 2 & 0 \\
\hline & Imprensa & 2 & 2 & 0 & 0 \\
\hline Experiência anterior negativa & & 5 & 4 & 1 & 0 \\
\hline $\begin{array}{l}\text { Doenças abrangidas pela vacina são pouco graves e não necessitam } \\
\text { de prevenção }\end{array}$ & & 3 & 0 & 2 & 1 \\
\hline \multirow[t]{4}{*}{ A vacina provoca doenças } & Sim & 3 & & & \\
\hline & Autismo & 1 & 1 & 0 & 0 \\
\hline & Coqueluche & 1 & 1 & 0 & 0 \\
\hline & Meningite & 1 & 0 & 1 & 0 \\
\hline Falta informação/recomendação por profissionais de saúde & & 3 & 2 & 0 & 1 \\
\hline Diferentes informações/recomendações por profissionais de saúde & & 2 & 0 & 1 & 1 \\
\hline Cônjuge/companheiro(a) recusou vacinação & & 1 & 0 & 1 & 0 \\
\hline
\end{tabular}

* Todos os casos de indicação pelo médico assistente foram relacionados à vacina BCG. 


\section{DISCUSSÃO}

Foi constatado um número reduzido de RV, ou seja, de não adesão dos pais às vacinas do PNV. Estes dados são a favor das conhecidas taxas elevadas de cobertura vacinal dos últimos anos em Portugal [6]. $\mathrm{O}$ conhecimento dos motivos de RV referidos pelos pais reforça o modelo de abordagem de hesitação em vacinar do grupo de trabalho SAGE da OMS $[11,14]$. Esses motivos integram-se nas influências contextuais (nível socioeconômico/cultural; meios de comunicação social), nas influências individuais e de grupo (crenças pessoais; opção por outros métodos de prevenção; desvalorização da vacinação e assunção como não prioritária; desconhecimento, interpretações erradas, desinformação) e questões relacionadas com as próprias vacinas (riscos e benefícios) $[10,14,16]$. O nível educacional mais elevado foi encontrado no ACeS onde a taxa de RV foi maior - Porto Ocidental, constituindo um achado concordante com dados publicados na literatura sobre esta problemática $[8,16]$.

A ideia de que as "vacinas não são prioridade", "são pouco seguras" e o "receio dos efeitos colaterais", motivos mais frequentemente invocados pelos pais, integram-se num dos três fatores determinantes do "modelo 3C's" da hesitação em vacinar - complacência [10]. Os outros dois determinantes deste modelo - conveniência e confiança - assumiram, neste grupo em estudo, uma importância relativamente inferior $[9,14]$. Por outro lado, a revelação de famílias com nível acadêmico elevado, com opção por "outros métodos naturais de prevenção doenças", "dieta específica" e "informação negativa sobre vacinas" poderão indicar comunidades alvo de maior risco de não vacinação, e, portanto, um potencial grupo para melhor caracterização. Outros motivos, como crenças pessoais e atitudes perante a vacinação, dúvidas acerca da eficácia, informação errada, e até informações discrepantes por profissionais de saúde, poderão revelar uma necessidade urgente de intervenção.

$O$ fato da informação prestada pelo médico assistente ter sido relacionada à vacina BCG foi consistente com a epidemiologia local da doença no período de realização do estudo. Assim, a informação pelo médico assistente da incidência decrescente da tuberculose em Portugal e dessa doença não constituir um problema de saúde pública poderá ter contribuído pela opção dos pais pela não vacinação dos seus filhos. Acrescenta-se ainda, um período de rotura do fornecimento da vacina BCG em Portugal em 2015 e, apesar de fora do âmbito temporal do atual estudo, o lançamento no início de 2016 de uma norma de vacinação com $\mathrm{BCG}$ exclusiva a grupos de risco em crianças com idade inferior a seis anos (Norma 001/2016 de 12/02/2016, revogada em 29/06/2016).

Uma limitação deste estudo é o fato de ter sido aplicado a uma população urbana e regional de Portugal, podendo não traduzir a realidade total do problema em nível nacional. Entretanto este trabalho pode ser considerado um estudo inovador, ao ter permitido o conhecimento do número e motivos de RV numa população urbana portuguesa, ampliando o conhecimento do problema da RV (integrado no grande grupo da hesitação em vacinar) e dos fatores envolvidos.

Nesta população foi pequena a taxa de RV; no entanto, com base nos resultados obtidos, poderão ser dirigidos esforços de intervenção junto às famílias, visando combater os argumentos sem base científica e obter uma adesão inequívoca ao PNV em Portugal. Essa intervenção poderá englobar a sensibilização de profissionais e da população em geral, acerca das vantagens das vacinas, importância e segurança da vacinação e doenças relacionadas, assim como da falta de evidência científica dos argumentos dos proponentes da não vacinação. A replicação deste estudo deverá ser considerada, para melhor caraterização do problema, generalização de resultados e definição das mais adequadas estratégias de intervenção.

\section{NOTAS}

\section{Agradecimentos}

Os autores agradecem aos pais que aceitaram participar no estudo, aos enfermeiros responsáveis pela vacinação das unidades de saúde dos $\mathrm{ACeS}$ incluídos no estudo, nomeadamente à Enf ${ }^{\mathrm{a}}$ Conceição Costa da USP do ACeS Ocidental, à Enf- Teresa Cardoso da USP do ACeS Matosinhos e à Enf. Margarida Vieira da Equipa de Coordenação Regional do PNV, por sua disponibilidade e colaboração no fornecimento de dados. Igualmente à Dr ${ }^{\mathrm{a}}$ Maria do Céu Ribeiro, agradecem a discussão inicial sobre o tema. À Dra ${ }^{\mathrm{a}}$ Ana Leça, pelo especial interesse e atenção dedicada ao presente trabalho.

Apoio financeiro

Este estudo não recebeu apoio financeiro de fontes externas.

Declaração de conflito de interesses

Os autores declaram não haver conflitos de interesses relevantes ao conteúdo deste estudo.

Contribuições dos autores

Todos os autores fizeram contribuições substanciais para concepção, ou delineamento, ou aquisição, ou análise ou interpretação de dados; e redação do trabalho ou revisão crítica; e aprovação final da versão para publicação.

Disponibilidade dos dados e responsabilidade pelos resultados

Todos os autores declaram ter tido total acesso aos dados obtidos e assumem completa responsabilidade pela integridade destes resultados. 


\section{REFERÊNCIAS}

1. World Health Organisation. Fact sheet: immunization coverage [Internet] [cited 2017 October 04]. Available from: http:// www.who.int/mediacentre/factsheets/fs378/en/

2. World Health Organization, United Nations International Children's Emergency Fund, The World Bank. State of the World's Vaccines and Immunizations. 3rd ed. Geneva, Switzerland: World Health Organization; 2009.

3. Centralized information system for infectious diseases (CISID) online database: World Health Organization Regional Office for Europe [Internet]. Copenhagen; 2014 [cited 2018 April 16]. Available from: http://data.euro.who.int/cisid/

4. Portugal. Direção-Geral da Saúde. Programa Nacional de Vacinação 2012, Norma 040/2011 DGS [Internet]. Lisboa; 2011 [updated 2012 Jan; cited 2018 April 8]. Available from: https://www.dgs.pt/directrizes-da-dgs/normas-e-circularesnormativas/norma-n-0402011-de-21122011-atualizada-a-26012012

5. Portugal. Direção-Geral da Saúde. Programa Nacional de Vacinação 2017, Norma 16/2016 DGS [Internet]. Lisboa; 2017 [updated 2017 Jul; cited 2018 April 10]. Available from: https://www.dgs.pt/directrizes-da-dgs/normas-e-circularesnormativas/norma-n-0162016-de-16122016.aspx

6. Portugal. Direção-Geral da Saúde. Programa Nacional de Vacinação - Avaliação 2015 DGS [Internet]. Lisboa; 2016 [updated 2016; cited 2018 April 10]. Available from: https://www.dgs.pt/documentos-e-publicacoes/boletim-vacinacaon-10-abril-de-2016-pdf.aspx

7. Andre FE, Booy R, Bock HL, Clemens J, Datta SK, John TJ: Vaccination greatly reduces disease, disability, death and inequity worldwide. Bull World Health Organ. 2008;86: 140-6. https://doi.org/10.2471/BLT.07.040089

8. Berezin M, Eads A. Risk is for the rich? Childhood vaccination resistance and a culture of health. Soc Sci Med. 2016;165:233-45. https://doi.org/10.1016/j.socscimed.2016.07.009

9. MacDonald NE. Vaccine hesitancy: Definition, scope and determinants. Vaccine. 2015;33:4161-4. https://doi.org/10.1016/j. vaccine.2015.04.036

10. Larson HJ, Jarret C, Eckersberger E, Smith D, Paterson P. Understanding vaccine hesitancy around vaccines and vaccination from a global perspective: A systematic review of published literature, 2007-2012. Vaccine. 2014;32: 2150-9. https://doi.org/10.1016/j.vaccine.2014.01.081

11. Favin M, Steinglass R, Fields R, Banerjee K, Sawhney M. Why children are not vaccinated: A review of the grey literature. Int Health. 2012;4:229-38. https://doi.org/10.1016/j.inhe.2012.07.004

12. Lim WY, Amar-Singhab HSS, Jeganathana N, Rahmatc H, Mustafa N.A, Mohd Yusof FS, Rahman R. Itam S, Chan CH, N-Julia MS. Exploring immunisation refusal by parents in the Malaysian context. Cogent Medicine. 2016;3(1):1142410. https://doi.org/10.1080/2331205X.2016.1142410

13. Ołpiński M. Anti-Vaccination Movement and Parental Refusals of Immunization of Children in USA. Pediatria Polska. 2012;87:381-5. https://doi.org/10.1016/j.pepo.2012.05.003

14. European Centre for Disease Prevention and Control. Rapid literature review on motivating hesitant population groups in Europe to vaccinate. Stockholm: ECDC; 2015.

15. Dubéa E, Gagnona D, Nickelsd E, Jeramd S, Schuster M. Mapping vaccine hesitancỹ Country-specific characteristics of a global phenomenon. Vaccine. 2014;32:6649-54. https://doi.org/10.1016/j.vaccine.2014.09.039

16. Larson HJ, Jarrett C, Eckersberger E, Smith D, Paterson P. Understanding vaccine hesitancy around vaccines and vaccination from a global perspective: A systematic review of published literature. Vaccine. 2014;32:2150-9. https:// doi.org/10.1016/j.vaccine.2014.01.081

17. Santos P, Hespanhol A. Recusa vacinal - o ponto de vista ético. Rev Port Med Geral Fam. 2013;29:328-33. https://doi. org/10.32385/rpmgf.v29i5.11167 